\title{
BMJ Open Managing the transition (ManTra): a resource for persons with secondary progressive multiple sclerosis and their health professionals: protocol for a mixed-methods study in Italy
}

\author{
Ambra Mara Giovannetti, ${ }^{1,2}$ Andrea Giordano, ${ }^{2}$ Erika Pietrolongo, ${ }^{3}$ \\ Paolo Confalonieri, ${ }^{1}$ Giovanna De Luca, ${ }^{3}$ Carla Tortorella, ${ }^{4}$ Maria Trojano, ${ }^{4}$ \\ Michele Messmer Uccelli, ${ }^{5}$ Valentina Torri Clerici, ${ }^{1}$ Lara Gitto, ${ }^{6}$ Sascha Köpke, ${ }^{7}$ \\ Claudia Borreani, ${ }^{8}$ Christoph Heesen, ${ }^{9}$ Alessandra Solari, ${ }^{2}$ on behalf of the ManTra \\ project
}

To cite: Giovannetti AM, Giordano A, Pietrolongo E, et al. Managing the transition (ManTra): a resource for persons with secondary progressive multiple sclerosis and their health professionals: protocol for a mixed-methods study in Italy. BMJ Open 2017;7:e017254. doi:10.1136/ bmjopen-2017-017254

- Prepublication history and additional material for this paper are available online. To view, please visit the journal (http:// dx.doi.org/10.1136/bmjopen2017-017254).

Received 12 April 2017 Revised 22 June 2017 Accepted 12 July 2017

CrossMark

For numbered affiliations see end of article.

Correspondence to Dr Alessandra Solari; alessandra.solari@istitutobesta.it

\section{ABSTRACT}

Introduction 15 years after clinical onset, about 50\% of patients with relapsing-remitting multiple sclerosis convert to secondary progressive multiple sclerosis (SPMS). Notwithstanding the importance of this transition, knowledge of the experiences and needs of patients and carers is fragmentary, and targeted interventions are not available. Managing the Transition to SPMS (ManTra) is a mixed methodology project to develop and test a user-led resource for newly diagnosed patients with SPMS. Here, we describe the developmental phase, consisting of a literature review and a new research study involving key stakeholders, in which we construct the resource. Methods and analysis Round 1: The literature review and study will be conducted in parallel. The latter will identify patient needs using a qualitative approach consisting of: personal semistructured interviews with $>15$ recently diagnosed patients with SPMS; three focus group meetings (one with significant others of patients, one with neurologists and one with other health professionals caring for patients with SPMS). An online survey (>200 recently diagnosed Italian patients with SPMS) will follow to verify needs in a larger independent sample. An expert panel will outline a set of candidate resources/interventions that aim to satisfy the needs thus identified. Round 2: Consensus on the final resource will be obtained in a 1-day meeting of recently diagnosed patients with SPMS, significant others, health professionals and other stakeholders, using the nominal group technique. The expert panel will refine the resource, identify the outcome measures to assess its efficacy and ascertain the most suitable comparator (ManTra Phase 2, not part of this protocol).

Ethics and dissemination The study protocol was approved by the ethics committees of each of the involved centres: Foundation IRCCS Neurological Institute C Besta, Milan ; G D'Annunzio University of Chieti-Pescara and the Aldo Moro University of Bari. The results will be published in peer-reviewed journals, presented at conferences and a lay summary sent to participants.

\section{Strengths and limitations of this study}

- The resource will be developed to respond to the needs and preferences of secondary progressive multiple sclerosis (SPMS) patients.

- The outcome measures to assess the efficacy of the resource in the future trial will be identified.

- Our understanding of the ways in which patients and their significant others experience the transition to SPMS will be improved.

- Study findings and deliverables will be for Italy, which may limit transferability to other contexts and healthcare systems.

\section{INTRODUCTION}

About $85 \%$ of patients with multiple sclerosis (MS) initially experience one or more relapses followed by complete or incomplete recovery. This is the relapsing-remitting (RR) phase of the disease. By about 15 years after clinical onset, around $50 \%$ of patients with RRMS have developed progressive disease $\left(\right.$ secondary progressive MS, SPMS ${ }^{1}$; median time to SPMS is consistently reported at around 20 years. ${ }^{1-4}$

SPMS is characterised by progressive accumulation of disability over at least 6 months after an initial RR course, with or without acute exacerbations during progression. ${ }^{5}$ Conversion from RRMS to SPMS is considered a key determinant of long-term disease prognosis. ${ }^{1}$ However, neither imaging criteria nor biomarkers are available to objectively distinguish RRMS from SPMS, and SPMS is still diagnosed retrospectively. ${ }^{6-8}$ In fact, the RRMS-SPMS transition is a period of diagnostic uncertainty that may last for several years (3years on average) ${ }^{9}$ 
The RRMS-SPMS transition is also critical from the psychosocial point of view. ${ }^{10}$ As well as new uncertainty, people with MS, their families and health professionals (HPs) involved in patient care all have to adjust to the new reality of unremitting symptoms and activity limitations and absence of effective disease-modifying treatments. ${ }^{11}$

Published studies have generally been concerned with patient and relative needs at the time of diagnosis ${ }^{12}{ }^{13}$; at the beginning of or during change of disease-modifying treatment ${ }^{14}$ and managing the disease in general. ${ }^{10}{ }^{15-20}$ Some studies on needs of people with severe MS have been also published recently. ${ }^{21-25}$ However, few studies have investigated the experiences of patients and their significant others (SO) around the RRMS-SPMS transition, ${ }^{26}$ and no studies have assessed ad hoc interventions for empowering and improving the quality of life of newly diagnosed patients with SPMS. This lack is particularly relevant because the period surrounding the transition may be as demanding as the disclosure of the MS diagnosis (for patients, SOs and HPs) ${ }^{27}$ Neurologists report that patients experience the transition as like 'being diagnosed again'. ${ }^{10}$ A person with SPMS commented, "Hearing I have a form of MS that is chronically progressive and has no therapies felt like being told to go home and make the best of it because they had nothing to offer me". ${ }^{11}$ Once a patient has changed to SPMS, the frequency of contact with a neurologist may decrease, with increased risk of feeling abandoned. ${ }^{12}$ These data suggest that patient management should change to meet the challenge of altered disease course. ${ }^{10} \mathrm{~A}$ recent qualitative study identified four main themes in relation to patient and carer experience of disease progression: 'realisation'-how patients become aware of having shifted to SPMS; 'reaction'-their response to this realisation; 'realities' of living with SPMS (dealing with the healthcare system during this period) and 'future challenges' envisaged by patients and carers. ${ }^{28}$ The same group also explored the experiences of HPs supporting patients during the transition. Two main themes were found: 'transition' which comprised issues related to recognising and communicating about SPMS and 'providing support' which included descriptions of challenging aspects of patient care, providing support for caregivers, using the multidisciplinary team and difficulties due to service limitations. ${ }^{29}$ Providing adequate psychological support and engaging patients with self-management approaches emerged as particularly challenging. ${ }^{29}$

\section{Theoretical framework}

The theoretical framework of the project is the biopsychosocial model, which supersedes the biomedical model and has more complex conception of a person's functioning. The model recognises disability as a relation between health status and the person's environment. ${ }^{30}$ Disability is conceptualised as a difficulty in functioning at the level of the body, the person or society, in one or more life domains, as experienced by an individual with a health condition in interaction with contextual factors. ${ }^{31}$ This framework guided the development of our protocol, for example, the decision to include different stakeholders so as to obtain a multilevel understanding of the phenomenon and as a way of taking personal and environmental factors into account when exploring patient needs and thinking about a resource. We intend no restriction as to the target of the resource (patient, patient environment). Furthermore, the definition of the resource itself will be influenced by the practical application of the model-'biopsychosocially oriented clinical practice', which Borrell-Carrió et al. described as based on the principles of calibrating with the physician, creating trust, cultivating curiosity, recognising bias, educating the emotions, using informed intuition and communicating clinical evidence. ${ }^{32}$

Consistent with the principles of the biopsychosocial model, we also refer to empowerment theory. Whitmore defined empowerment based on the following assumptions: (1) individuals are assumed to understand their own needs better than anyone else and should therefore have the power both to define and act on them; (2) all people possess strengths on which they can build; (3) empowerment is a lifelong endeavour; (4) personal knowledge and experience are valid and useful for effective coping. ${ }^{33}$ Supporting people with SPMS in their quest to attain personal empowerment helps them optimise their inner strengths and better interact with their environment, to hence achieve improved quality of life. Consistent with the biopsychosocial model, empowerment theory drives interventions aimed at improving interactions between people and their environment, encouraging the potential for growth.

The transition to SPMS is a new and challenging phase for patients with MS, with no established treatment options to delay or prevent further worsening and major issues arising regarding personal and working life. Improving the communication skills of neurologists and their ability to elicit patient views, concerns and preferences in the context of managing (and self-managing) the SPMS transition is vital. ${ }^{34-39}$

To do this, it is essential to thoroughly investigate what happens during the transition. It is equally important to use reliable measures to capture SPMS experiences of care and find ways of translating findings into improvements that render services more responsive to patient needs and preferences. ${ }^{40}$ It is for these reasons that we conceived the Managing the Transition (ManTra) project. It has two aims: (1) to assess the experiences and the needs of people recently converted to SPMS, using qualitative and quantitative research and involving key stakeholders and (2) to set up a user-led resource ${ }^{41}$ to empower and improve the quality of life of newly diagnosed patients with SPMS. The resource can be directed to the patient or the patient environment.

\section{METHODS}

ManTra is a multicentre study employing mixed methods and adhering to the Medical Research Council (MRC) 


\section{ManTra Flow Chart}

Phase 1: Development of the resource

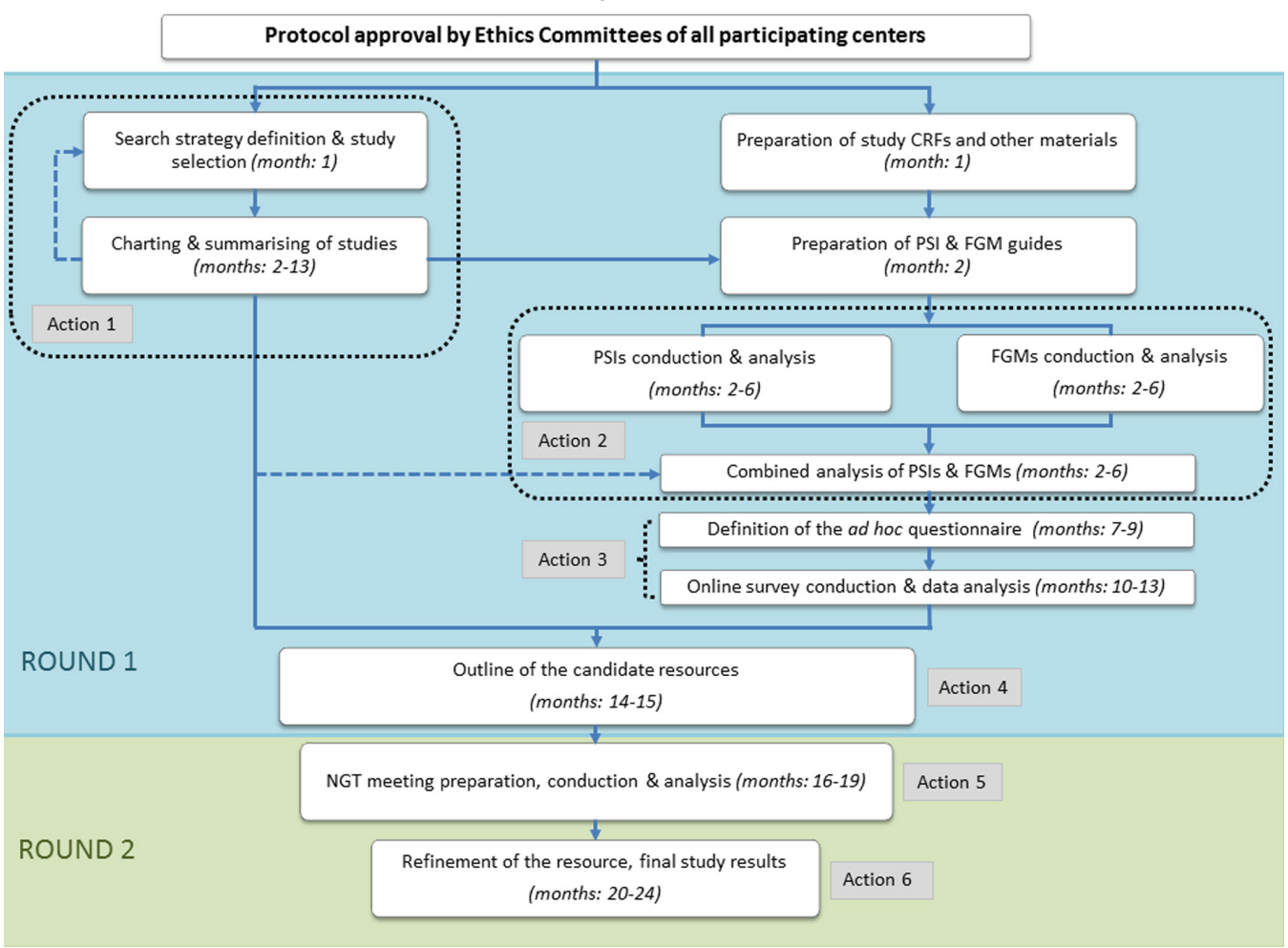

Figure 1 Flow chart of the developmental phase of the ManTra project. CRF, case report form; FGM, focus group meeting; ManTra, Managing the Transition project; NGT, nominal group technique; PSI, personal (one-to-one) semistructured interview.

framework for developing and evaluating complex interventions. ${ }^{42}$ The development phase presented here comprises six main actions grouped into two rounds (figure 1).

In round 1 , we will survey the literature and at the same time assess patient needs by means of personal (one-to-one) semistructured interviews (PSIs) with patients and focus group meetings (FGMs) with key stakeholders (SPMS patient SOs, treating neurologists and other HPs, including psychologists, nurses, social workers, psychiatrists and therapists). We selected PSIs so as to provide an environment conducive to recounting personal experiences and emotions, whose setting and duration could be adjusted to the needs of the individual patient (eg, fatigue and other MS symptoms). We opted for FGMs for stakeholders as they examine multiple perspectives simultaneously, and promote interaction, brainstorming and elaboration of ideas.

Findings from actions 1 and 2 will be used to construct an online patient survey, allowing us to verify whether the needs identified in PSIs are pertinent to a larger sample of people with SPMS and to reveal additional issues. Based on the integrated findings, an expert panel will define candidate resources for newly diagnosed people with SPMS.

In round 2, consensus on the resource will be achieved using the nominal group technique. The expert panel will refine the resource and identify outcome measures and the most suitable comparator for assessing its effectiveness.

\section{Round 1}

Action 1: Literature review

Following the Arksey and O'Malley framework, ${ }^{43}$ further refined by Levac, ${ }^{44}$ we will perform a scoping study to: (1) map the existing literature on the transition to SPMS; (2) examine the extent, range and nature of the available evidence; (3) summarise findings as a guide to subsequent project actions.

We will assess clinical trials, qualitative studies and observational studies concerned with the RRMS-SPMS transition. We are particularly interested in identifying/ developing resources to empower and support patients with SPMS, SOs and HPs. Studies providing information potentially useful for developing such resources will be also accessed. Our database search (inception to March 2017; no language restriction) will be applied to Medline (see online supplementary appendix) and adapted for EMBASE, PsycINFO, CINAHL and Google Scholar. We will also search the PROSPERO registry (for systematic review protocols) and the Cochrane Library (for systematic reviews). We will check the following sources for unpublished, ongoing or additional studies: trial and dissertation registries (http://apps.who.int/trialsearch; https://www.isrctn.com; https://clinicaltrials.gov/ct2/ home); reference lists of selected studies; key textbooks 
and conference proceedings (http://apps.isiknowledge. com). Finally, we will contact experts in the field and investigators working in this area to obtain unpublished data or grey literature.

Two researchers will check titles and abstracts identified by the search and decide independently which should be examined further. As recommended, ${ }^{4344}$ criteria selecting studies for further appraisal will be devised after scoping the initial findings. All potentially relevant studies will be obtained in full text and assessed independently for inclusion. Studies that do not meet inclusion criteria will be excluded and the reasons for exclusion documented. Disagreements about inclusion will be resolved by discussion, if necessary with the help of a third researcher. Researchers will not be masked to study author identity. We will develop a data-charting form for reporting the following information about included studies: author(s); year of publication; study location; population; intervention (if any); intervention components (using the TiDiER framework, http://www.equator-network.org/reportingguidelines/tidier/); study aims; methodology; outcome measures and main findings.

The reporting of process-related outcomes will be assessed using the revised Criteria for Reporting the Development and Evaluation of Complex Interventions in Healthcare. ${ }^{45}$ We will assess the quality of the included studies using the Cochrane risk of bias tool for randomised controlled trials, ${ }^{46}$ and pertinent checklists of the Critical Appraisal Skills Programme for other study designs (http://www.casp-uk.net/casptools-checklists). Quality appraisal will be incorporated into the synthesis process either narratively or through sensitivity analysis.

In accordance with the concept of scoping studies, ${ }^{43} 44$ no formal data synthesis will be performed: we instead will provide basic data on the extent, nature and distribution of the included studies. We will also summarise results as a framework, themes or list of findings, as appropriate.

The results of this action will contribute to defining the qualitative study (Action 2) and will inform the content of the candidate resources (Action 3).

\section{Action 2: Qualitative study on experiences and needs of people} involved with the RRMS-SPMS transition

The experiences and needs of patients transitioning to SPMS will be explored by PSIs with recently diagnosed patients with SPMS, and by FGMs involving patient SOs, neurologists and other HPs. PSI and FGM guides will be developed in a 3-day seminar chaired by $\mathrm{CB}$, a psychology expert in qualitative research. AG, AMG, EP and CB will be in charge of devising the guides, defining questions and prompts so as to maximise the possibility of obtaining high-quality information from the different stakeholders. Up to 15 other researchers interested in the topic will also participate, with the purpose of promoting discussion. The seminar will cover the following:

Day 1. ManTra study overview; introduction to qualitative research methods; results of the scoping review; principles of interview guide development; peer discussion; construction of PSI guides.

Day 2. Review of PSI guides; introduction to holding FGMs; principles of FGM guide development; peer discussion; construction of FGM guides for neurologists and HPs.

Day 3. Review of FGM guides for neurologists and HPs; construction of FGM guides for patient SOs; peer discussion; review of the FGM guide for patient SOs.

Investigators from each participating Italian centre will identify potentially eligible patients from the MS centre database and invite them (and their SOs) to join the study. Potentially eligible MS neurologists and other HPs will be identified by the ManTra steering committee from Italian hospitals, rehabilitation centres and health centres, and invited to participate.

PSI and FGM participants will be selected using a purposive sampling technique. To cover a range of experiences, participants will be from the three geographic areas of Italy (North, Centre, South), and varied in terms of gender, education and (for patients) neurological compromise (Expanded Disability Status Scale ${ }^{47}$ ). PSIs and FGMs will be audiorecorded and transcribed in full.

A minimum of 15 PSIs will be held (five from each centre), with sampling continuing until no new themes emerge from the data (data saturation). ${ }^{48}$

Patients will be included if they:

- Are $\geq 18$ years of age;

- Were diagnosed with $\mathrm{SPMS}^{49} 3$ months to 5 years before inclusion;

- Are fluent in Italian;

- Gave written consent.

Patients will be excluded if they:

- Have severe cognitive compromise (according to the referring neurologist);

- Are unable to communicate effectively.

PSIs will be one-to-one meetings between a patient and a trained interviewer that take place in a dedicated room at an MS centre or exceptionally at a patient's home. Estimated duration is about $30 \mathrm{~min}$, with a maximum of an hour. Before the interview starts, patients will complete the COSM-S questionnaire ${ }^{50}$ in a version adapted (minor changes) to the communication of SPMS diagnosis. The interviewer will explain the purpose of the interview and then pose the questions. Patients will be encouraged to elaborate on themes that emerge and give their own views.

Three FGMs will be held in round 1: one each for SOs, neurologists and other HPs concerned with people with SPMS. SOs will be relatives, partners or close friends of the patient who received a diagnosis of $\mathrm{SPMS}^{47}$ in the 3 months to 5 years prior to inclusion. SOs will be included if they:

- Are $\geq 18$ years of age;

- Provided emotional or tangible support to the patient during the SPMS disclosure period ${ }^{51}$;

- Are fluent in Italian; 
Gave written consent.

SOs will be excluded if they have overt cognitive compromise or any impairment preventing effective communication. We will make efforts to include SOs of SPMS patients with severe cognitive compromise.

Neurologists and other HPs will be eligible if they are experienced in caring for SPMS patients, fluent in Italian and provide written informed consent.

Each FGM will have 6 to 10 participants plus two moderators. One moderator (facilitator) will engage all participants, promoting exchange, modulating conflicts, ensuring the topics are adequately covered and allowing sufficient time for exploration of pertinent issues arising. The facilitator will first explain the purpose of the meeting, get participants to introduce themselves, then guide the meeting, introducing each topic in turn. After discussion of all scheduled topics, the facilitator will summarise major points arising and ask for further feedback. The comoderator will take notes, record relevant non-verbal communication, oversee the audio recording and otherwise assist as necessary.

\section{Action 3: Online survey}

The experiences and needs of recently diagnosed patients with SPMS will be further assessed by an online survey (dedicated website) of a minimum of 200 patients. To increase the external validity of the data, recruitment will be extended to the other Italian MS centres (around 250) and will involve the MS Study Group of the Italian Neurological Society (SIN) and the Italian MS Society (AISM). The survey will close after 2 months, or after 250 patients have contributed but will be extended to 4 months if 200 surveys are not completed in the first 2 months. The referring neurologist will identify potentially eligible patients for the online survey from the MS centre database and invite them to participate by contacting the ManTra coordinating unit (email and phone contact will be provided).

The coordinating unit will verify patient eligibility (same eligibility criteria as for PSIs except that patients with severe visual or other impairment precluding completion of the survey will be also excluded). Eligible patients will be given the credentials to access the Survey Monkey platform. The platform will be password protected to ensure patient data protection and also to prevent patients from completing the questionnaires more than once. No incentives (monetary or non-monetary) will be offered to survey participants.

The online survey will include three questionnaires presented in the following order: (1) a brief sociodemographic and clinical questionnaire (requiring about $5 \mathrm{~min}$ to complete); (2) the COSM-S, in a version adapted to the communication of the SPMS diagnosis (about $15 \mathrm{~min}$ to complete) $)^{50}$ and (3) an ad hoc questionnaire to be devised based on the results of actions 1 and 2 (completion time not to exceed $20 \mathrm{~min}$ ). Before fielding the survey, the usability and functionality of the three questionnaires will be tested on five patients with MS .
COSM-S is a patient self-assessed questionnaire probing satisfaction with the moment of MS diagnosis disclosure (section 1, five items) and the following period (section 2, 12 items satisfying Rasch model expectations). ${ }^{50}$ An expert panel produced COSM-S based on the Doctor's Interpersonal Skills Questionnaire, ${ }^{52}$ a literature review and patient interviews. ${ }^{53}$ The instrument has good internal consistency, test-retest reliability ${ }^{53}$ and responsiveness. ${ }^{545}$ We will use a modified COSM-S since tools assessing patient experience of the RRMS-SPMS transition are not available, COSM-S is useful for identifying where improvements are needed during MS diagnosis disclosure and may be appropriate in other clinical situations after minor modification. ${ }^{53}$

Based on findings of the literature review, the qualitative study and the online survey, the expert panel will outline the candidate resources. The panel consists of a researcher (health economist) who has had MS for 15 years (LG), a psychologist/methodologist (AG), two neurologists (CT, VT), two MS psychologists (AMG, EP) and a nurse/AISM delegate (MMU). A maximum of four resources will be proposed (Action 4), each sufficiently detailed to make assessment in Action 5 possible (see round 2 ).

\section{Round 2}

The nominal group technique will be used to compare and prioritise the resources outlined in round 1.5657 There will be a 1-day meeting with 16 to 20 participants recruited from the following categories (four to five persons each):

a. SPMS patients from three geographic areas of Italy (same eligibility criteria as for PSIs);

b. SOs of SPMS patients (same eligibility criteria as for SO FGM);

c. Neurologists and other HPs caring for SPMS patients;

d. Health service researchers and representatives of Italian patient and citizen organisations.

Two weeks before the meeting, each participant will receive an information pack detailing the candidate resources. Written informed consent will be obtained from participants at the meeting which will be held at the Foundation IRCCS Neurological Institute C. Besta, Milan and will be audio-recorded and transcribed verbatim. It will be structured as follows:

- Plenary session 1: The moderator will explain meeting's purpose and phases, the criteria to be used to prioritise the candidate resources, the importance of each participant's contribution and ask participants to introduce themselves. The moderator will then describe each candidate resource.

- Group sessions: Participants will be divided into four uniform groups (groups a-d reported above) to facilitate going over the nominal group process in a group that feels on an equal footing with each other. Each group will discuss the resources guided by a facilitator. Each participant will then be asked to rank the candidate resources for relevance, appropriateness and ease of implementation. 
- Plenary session 2: The priority ranking (overall and at the group level) will be presented, and the most suitable resource identified based on overall priority ranking. Ideas and comments useful for resource refinement will be discussed and noted.

The ManTra expert panel will refine the resource selected in plenary session 2 . It will identify the outcome measures (quality of life and other pertinent measures such as health locus of control, self-efficacy and care satisfaction) and the most suitable comparator (eg, usual care or low intensity intervention) to assess the effectiveness of the resource.

\section{ANALYSES}

\section{Statistical analysis (online survey)}

We based our sample size on the COSM-S section 2 score. ${ }^{50}$ We estimate (one-sample comparison) that 197 patients are sufficient to detect, with a power of 0.80 , a mean score of 40 (SD 10), compared with a hypothesised mean population score of 38 , at a two-sided alpha level of 0.05 .

For the results of the three online questionnaires, categorical variables will be summarised as counts and percentages, and compared by $\chi^{2}$ or Fisher's exact test, as appropriate; continuous variables will be summarised as means and SD or medians with interquartile ranges. Normality and equality of variance assumptions will be tested using Shapiro-Wilk's and Bartlett's tests, respectively. Correlations will be assessed with Spearman's rho.

Preliminary univariate linear regression analyses will be used to assess which individual independent variables predict patient satisfaction (COSM-S section 2 score). Variables tested will be: age, sex, education, disease duration, disease severity and area of Italy. A multivariate linear stepwise regression model, using a mix of forward selection and backward elimination, will then be used to test a weighted combination of variables as significant predictors of patient satisfaction. For this model, we will assess the distribution of residuals for normality (Shapiro-Wilk test), heteroscedasticity (Breusch-Pagan test) and also check for multicollinearity (variance inflation factor).

Ad hoc questionnaire: For frequently reported ( $\geq 20 \%$ of participants) needs, logistic regression models will be used to investigate associations between each need and the following independent variables: age, sex, education, disease duration, disease severity, COSM-S scores and area of Italy. Continuous independent variables will be dichotomised with medians as cut-offs. The goodness of fit of the model will be investigated with the Hosmer-Lomeshow test. Results will be reported as ORs with 95\% CIs. The analyses will be performed with Stata Statistical Software, V.12. All statistical tests will be two tailed; differences will be considered significant at an alpha level of 0.05 .

\section{Qualitative analysis}

The methods of framework analysis will be applied to the PSI and FGM transcripts. Framework analysis uses an inductive approach to identify, extract and analyse core themes. ${ }^{485859}$ The transcripts will be analysed in six successive steps (see below), each of which embodies an increasing level of generalisation. ${ }^{60}$ To enhance the validity of this process, two researchers will analyse the transcripts independently (FGMs, steps 1-4; PSIs, steps $1-5)$ and jointly (step 6).

Steps in the analysis:

1. The researcher identifies all propositions considered significant, without considering their relation to other parts of the transcript and appends comments to these significant propositions.

2. Comments are expanded and contextualised along with the entire PSI/FGM.

3. Relations between comments are established by reordering and regrouping them by subject.

4. Themes are extrapolated and hierarchically ordered into categories, moving from general concepts to more specific ones.

5. Each PSI transcript analysis is compared with the others to identify common themes and also differences.

6. The analyses produced by the two researchers are compared, and a consensus is arrived at.

When the analysis of the first 15 PSIs (five from each centre) is completed, subsequent interviews will be analysed sequentially, and the researchers will decide to stop doing PSIs when no new themes arise (data saturation). ${ }^{48}$

A report of each FGM analysis will be submitted to FGM participants for review (respondent validation). In a later meeting, the researchers will produce a joint report that integrates findings from the PSI and FGM analyses; the joint report will also contain a structured précis of the data.

The transcripts of the nominal group meeting will be analysed by two researchers using thematic analysis ${ }^{585}$ to describe and compare the views of the stakeholders on the candidate resources. A report of this analysis will be submitted to meeting participants for review.

\section{ETHICS AND DISSEMINATION}

The study has been given ethical approval by the ethics committees of the Foundation IRCCS Neurological Institute C Besta (internal reference numbers 27, 29), the G d'Annunzio University of Chieti-Pescara (internal reference number 19,3/11/2016) and the Aldo Moro University of Bari (internal reference number $98793 \mathrm{CE}$, $30 / 12 / 2016)$. The results will be published in peer-reviewed journals, presented at conferences and a lay summary will be sent to participants. 


\section{DISCUSSION}

The development phase of ManTra aims to assess the experiences and the needs of people involved in the RRMS-SPMS transition and hence develop a user-led resource for empowering and improving the quality of life of newly diagnosed people with SPMS. The study is organised into two rounds. In the first, we will review the literature on the RRMS-SPMS transition by means of scoping study and assess stakeholder needs using mixedmethods research. This round's deliverables will be (up to) four candidate resources for newly diagnosed patients with SPMS .

In the second round, the nominal group technique will be used to achieve a stakeholder consensus on which resource to adopt. The expert panel will refine the resource and identify which outcome measures and comparator should be used to assess its efficacy in a future randomised controlled trial.

To our knowledge, this is the first study aimed at creating a user-led resource to promote empowerment and improve the quality of life of newly diagnosed patients with SPMS. We believe the MRC framework will ensure a 'bottom up' development, and a theory-driven intervention, so that the subsequent trial will not lack appropriate theory and ground work. ${ }^{42}$ Furthermore, integrated use of qualitative and quantitative methods and a multiple stakeholder perspective will maximise possibilities for extensive exploration of patient needs. When used in combination, qualitative and quantitative methods complement each other to afford a more exhaustive analysis (in ManTra these two methods will be integrated using a pragmatic approach). Mixed-methods research is now showing that it can make an important contribution to development of interventions and outcome measures which meet the values, preferences and needs of patients, their SOs and HPs. ${ }^{61}$ Our protocol will follow a rigorous qualitative methodology in line with Consolidated Criteria for Reporting Qualitative Research requirements (COREQ). ${ }^{62} 63$ The online survey will be designed following the Checklist for Reporting Results of Internet E-Surveys checklist (CHERRIES) ${ }^{64}$

There is a strong relationship between engagement of stakeholders in the planning and design of a study and subsequent quality and outcomes. ${ }^{65}$ Importantly, stakeholders will be involved not only in the appraisal of needs (round 1) but in resource construction and prioritisation. The nominal group technique is a recognised method of guiding consensus achievement ${ }^{56}$ that maximises informed group benefits and minimises drawbacks, chiefly domination by opinion makers and personal or group interests. The ManTra protocol has been recently adopted (ethical approval stage) by a team of German researchers that has collaborated long term with the Italian team in the area of shared decision-making. ${ }^{.738}$

\section{Expected benefits}

We expect that this project will provide an extensive insight into the delicate RRMS-SPMS transition and explore all stakeholders' experiences and views. We will develop an informed set of resource priorities and bring them to the attention of the scientific community. A new user-led resource will be designed and tested in a subsequent randomised controlled study.

\section{Limitation and concerns}

Although expect that needs and priorities of persons with SPMS will be similar across countries, particularly those with a healthcare system funded primarily by taxation, we do not expect our findings to be immediately generalisable outside Italy since health system structure and organisation varies considerably. In Italy, for example, there are wide differences in healthcare quality indicators and health expenditure between regions, with a clear cut north-south divide. ${ }^{66}$

Another possible limitation is the bias may be introduced by excluding patients with severe cognitive compromise or impairments preventing communication. It was considered necessary to exclude such patients to obtain reliable information and to avoid patient distress. However, we intend to include SOs of patients with severe cognitive compromise in the SO FGM so that we can obtain their views and hopefully incorporate their perspectives in the construction of the candidate resources. Bias could also be introduced by excluding from the online survey patients with severe visual impairment or any impairment precluding use of a computer.

Notwithstanding the advantages of the mixed methods approach, action planning, data collection and integration can be complex and therefore challenging. ${ }^{67}$ To limit these problems, the planned actions are highly coordinated, and the multidisciplinary research team (ManTra expert panel) will be supported during the project by findings from the literature review and the qualitative and quantitative analyses. The Italian MS Foundation will provide support and commitment that will facilitate appropriate allocation of resources and on time project completion.

\section{Author affiliations}

${ }^{1}$ Unit of Neuroimmunology and Neuromuscular Diseases, Foundation IRCCS Neurological Institute C Besta, Milano, Italy

${ }^{2}$ Unit of Neuroepidemiology, Foundation IRCCS Neurological Institute C Besta, Milano, Italy

${ }^{3}$ Department of Neuroscience, Imaging and Clinical Sciences, G d'Annunzio University of Chieti-Pescara, Chieti, Italy

${ }^{4}$ Department of Basic Medical Sciences, Neuroscience and Sense Organs, Aldo Moro University of Bari, Bari, Italy

${ }^{5}$ Italian Multiple Sclerosis Society and Research Foundation, Genova, Italy ${ }^{6}$ CEIS Economic Evaluation and HTA, Università degli Studi di Roma 'Tor Vergata', Roma, Italy

${ }^{7}$ Nursing Research Unit, University of Lübeck, Institute of Social Medicine and Epidemiology, Lübeck, Germany

${ }^{8}$ Unit of Clinical Psychology, Foundation IRCCS Istituto Nazionale per la Cura dei Tumori, Milano, Italy

${ }^{9}$ Department of Neurology, University Medical Center Hamburg-Eppendorf, Hamburg, Germany

Acknowledgements The authors thank Don Ward for help with the English and Drs Freya Davies and Abigail Methley for their critical but sympathetic reviews of our manuscript. 
Collaborators ManTra project investigators Steering Committee: CB, PC, GDL, AG, AMG (study Co-PI), L G, AS (study PI), VTC, MT, MMU. Literature review panel: A Fittipaldo, SK, AMG, AG. Qualitative analysis panel: CB, E Bianchi, AMG, EP. Expert panel: AG, AMG, LG, EP, MMU, V TC, C Tortorella. Centres and investigators: Foundation IRCCS Neurological Institute C Besta, Milan: Unit of Neuroepidemiology, AS, AG, G Ferrari, A Fittipaldo; Unit of Neuroimmunology and Neuromuscular Diseases: PC, AMG, VTC, S Rossi, R Mantegazza; Department of Neuroscience, Imaging and Clinical Sciences, G d'Annunzio University of Chieti-Pescara, Chieti: M Onofrj, EP, D Farina, D Travaglini, G DeLuca; Departments of Basic Medical Sciences, Neurosciences and Sense Organs, Aldo Moro University of Bari, Bari: MT, CT, E Luciannatelli, R Viterbo; Unit of Psychology, Foundation IRCCS Istituto Nazionale per la Cura dei Tumori, Milan: CB, E Bianchi; Italian Multiple Sclerosis Society and Research Foundation (AISM), Department of Health Services and Research, Genova: MMU; CEIS Economic Evaluation and HTA, Università degli Studi di Roma 'Tor Vergata', Rome, Rome: LG.

Contributors $A M G$ and $A S$ conceived and developed the study protocol. CB contributed expertise to the design of the qualitative study. AG and SK contributed expertise design of the scoping review. All authors contributed to the refinement of the study protocol. AMG and AS drafted the manuscript. All authors approved the final manuscript.

Funding This study is supported by the Fondazione Italiana Sclerosi Multipla (FISM, www.aism.it, grant $N^{\circ} 2015 / R / 22$ to AS). The funding source had no role in study design, data collection, data analysis, data interpretation or report writing.

Competing interests AS has been a board member of Biogen Idec, Merck Serono and Novartis; she has received speaker honoraria from Almirall, Excemed, Merck Serono, Sanofi Genzyme and Teva. CT has received speaker honoraria from Biogen, Merck Serono, Novartis, Genzyme, Teva, Almirall, Schering and Excemed.

Ethics approval The Ethic Committee of the Foundation IRCCS Neurological Institute C Besta.

Provenance and peer review Not commissioned; externally peer reviewed.

Open Access This is an Open Access article distributed in accordance with the Creative Commons Attribution Non Commercial (CC BY-NC 4.0) license, which permits others to distribute, remix, adapt, build upon this work non-commercially, and license their derivative works on different terms, provided the original work is properly cited and the use is non-commercial. See: http://creativecommons.org/ licenses/by-nc/4.0/

(C) Article author(s) (or their employer(s) unless otherwise stated in the text of the article) 2017. All rights reserved. No commercial use is permitted unless otherwise expressly granted.

\section{REFERENCES}

1. Scalfari A, Neuhaus A, Daumer M, et al. Onset of secondary progressive phase and long-term evolution of multiple sclerosis. J Neurol Neurosurg Psychiatry 2014;85:67-75.

2. Vukusic S, Confavreux C. Prognostic factors for progression of disability in the secondary progressive phase of multiple sclerosis. J Neurol Sci 2003;206:135-7.

3. Debouverie M, Louis S, Pittion-Vouyovitch S, et al. Multiple sclerosis with a progressive course from onset in Lorraine-Eastern France. $J$ Neurol 2007;254:1370-5.

4. Koch M, Kingwell E, Rieckmann P, et al. The natural history of secondary progressive multiple sclerosis. J Neurol Neurosurg Psychiatry 2010;81:1039-43.

5. Lublin FD, Reingold SC, Cohen JA, et al. Defining the clinical course of multiple sclerosis: the 2013 revisions. Neurology 2014;83:278-86.

6. Rovaris M, Confavreux C, Furlan R, et al. Secondary progressive multiple sclerosis: current knowledge and future challenges. Lancet Neurol 2006;5:343-54.

7. Pasquali L, Lucchesi C, Pecori C, et al. A clinical and laboratory study evaluating the profile of cytokine levels in relapsing remitting and secondary progressive multiple sclerosis. J Neuroimmunol 2015;278:53-9.

8. Dickens AM, Larkin JR, Griffin JL, et al. A type 2 biomarker separates relapsing-remitting from secondary progressive multiple sclerosis. Neurology 2014;83:1492-9.

9. Katz Sand I, Krieger S, Farrell C, et al. Diagnostic uncertainty during the transition to secondary progressive multiple sclerosis. Mult Scler 2014;20:1654-7.

10. Deibel F, Edwards M, Edwards A. Patients', carers' and providers' experiences and requirements for support in self-management of multiple sclerosis: a qualitative study. Eur J Pers Cent Healthc 2013;1:457-67.

11. Coetzee T, Zaratin P, Gleason TL. Overcoming barriers in progressive multiple sclerosis research. Lancet Neurol 2015;14:132-3.

12. Johnson $\mathrm{J}$. On receiving the diagnosis of multiple sclerosis: managing the transition. Mult Scler 2003;9:82-8.

13. Solari A. Effective communication at the point of multiple sclerosis diagnosis. Mult Scler 2014;20:397-402.

14. Heesen C, Solari A, Giordano A, et al. Decisions on multiple sclerosis immunotherapy: new treatment complexities urge patient engagement. J Neurol Sci 2011;306:192-7.

15. Forbes A, While A, Taylor M. What people with multiple sclerosis perceive to be important to meeting their needs. $J$ Adv Nurs 2007;58:11-22.

16. Koopman WJ, Benbow CL, Vandervoort M. Top 10 needs of people with multiple sclerosis and their significant others. J Neurosci Nurs 2006;38:369-73.

17. Lorefice L, Mura G, Coni G, et al. What do multiple sclerosis patients and their caregivers perceive as unmet needs? BMC Neurol 2013;13:177.

18. Ytterberg C, Johansson S, Gottberg K, et al. Perceived needs and satisfaction with care in people with multiple sclerosis: a two-year prospective study. BMC Neurol 2008;8:36

19. Ponzio M, Tacchino A, Zaratin P, et al. Unmet care needs of people with a neurological chronic disease: a cross-sectional study in Italy on multiple sclerosis. Eur J Public Health 2015;25:775-80.

20. Giovannetti AM, Brambilla L, Torri Clerici V, et al. Difficulties in adjustment to multiple sclerosis: vulnerability and unpredictability of illness in the foreground. Disabil Rehabil 2017;39:1-7.

21. Borreani C, Bianchi E, Pietrolongo E, et al. Unmet needs of people with severe multiple sclerosis and their carers: qualitative findings for a home-based intervention. PLoS One 2014;9:e109679.

22. Golla H, Galushko M, Pfaff $\mathrm{H}$, et al. Unmet needs of severely affected multiple sclerosis patients: the health professionals' view. Palliat Med 2012;26:139-51.

23. Galushko M, Golla H, Strupp J, et al. Unmet needs of patients feeling severely affected by multiple sclerosis in Germany: a qualitative study. J Palliat Med 2014;17:274-81.

24. Edmonds P, Vivat B, Burman R, et al. 'Fighting for everything': service experiences of people severely affected by multiple sclerosis. Mult Scler 2007:13:660-7.

25. Giovannetti AM, Pietrolongo E, Giordano A, et al. Individualized quality of life of severely affected multiple sclerosis patients: practicability and value in comparison with standard inventories. Qual Life Res 2016;25:2755-63.

26. Methley AM, Chew-Graham C, Campbell S, et al. Experiences of UK health-care services for people with multiple sclerosis: a systematic narrative review. Health Expect 2015;18:1844-55.

27. Thorne S, Con A, McGuinness L, et al. Health care communication issues in multiple sclerosis: an interpretive description. Qual Health Res 2004;14:5-22.

28. Davies F, Edwards A, Brain K, et al. 'You are just left to get on with it': qualitative study of patient and carer experiences of the transition to secondary progressive multiple sclerosis. BMJ Open 2015;5:e007674.

29. Davies F, Wood F, Brain KE, et al. The transition to secondary progressive multiple sclerosis: an exploratory qualitative study of health professionals' experiences. Int J MS Care 2016;18:257-64.

30. World Health Organization. The international classification of functioning, disability and health: ICF. Geneva: World Health Organization, 2001.

31. Leonardi M, Bickenbach J, Ustun TB, et al. MHADIE Consortium. The definition of disability: what is in a name? Lancet 2006;368:1219-21.

32. Borrell-Carrió F, Suchman AL, Epstein RM. The biopsychosocial model 25 years later: principles, practice, and scientific inquiry. Ann Fam Med 2004;2:576-82.

33. Whitmore E. Participation, empowerment and welfare. Canadian Review of Social Policy 1988;22:51-60.

34. Barratt $A$. Evidence based medicine and shared decision making: the challenge of getting both evidence and preferences into health care. Patient Educ Couns 2008;73:407-12.

35. Mulley AG, Trimble C, Elwyn G. Stop the silent misdiagnosis: patients' preferences matter. BMJ 2012;345:e6572.

36. Oshima Lee E, Emanuel EJ. Shared decision making to improve care and reduce costs. N Engl J Med 2013;368:6-8.

37. Pietrolongo E, Giordano A, Kleinefeld M, et al. Decision-making in multiple sclerosis consultations in Italy: third observer and patient assessments. PLoS One 2013;8:e60721.

38. Heesen C, Köpke S, Solari A, et al. Patient autonomy in multiple sclerosis-possible goals and assessment strategies. J Neurol Sci 2013;331:2-9. 
39. Solari A, Giordano A, Kasper J, et al. Role preferences of people with multiple sclerosis: image-revised, computerized self-administered version of the control preference scale. PLoS One 2013;8:e66127.

40. Coulter A. Can patients assess the quality of health care? BMJ 2006;333:1-2.

41. Rose D. Patient and public involvement in health research: ethical imperative and/or radical challenge? J Health Psychol 2014;19:149-58.

42. Craig P, Dieppe P, Macintyre S, et al. Medical research council guidance. Developing and evaluating complex interventions: the new medical research council guidance. BMJ 2008;337:a1655.

43. Arksey $\mathrm{H}, \mathrm{O}^{\prime}$ Malley L. Scoping studies: towards a methodological framework. Int J Soc Res Methodol 2005;8:19-32.

44. Levac D, Colquhoun H, O'Brien KK. Scoping studies: advancing the methodology. Implement Sci 2010;5:69.

45. Möhler R, Köpke S, Meyer G. Criteria for reporting the development and evaluation of complex interventions in healthcare: revised guideline (CReDECI 2). Trials 2015;16:204.

46. The Cochrane Collaboration. In: Higgins JPT, Green S, eds. Cochrane handbook for systematic reviews of interventions version 5.1.0. 2011.

47. Kurtzke JF. Rating neurologic impairment in multiple sclerosis: an expanded disability status scale (EDSS). Neurology 1983;33:1444-52.

48. Denzin NK, Lincoln YS, eds. Handbook of qualitative research. London, UK: Sage, 2000.

49. Polman $\mathrm{CH}$, Reingold SC, Banwell B, et al. Diagnostic criteria for multiple sclerosis: 2010 revisions to the McDonald criteria. Ann Neurol 2011;69:292-302.

50. Solari A, Grzeda M, Giordano A, et al. Use of Rasch analysis to refine a patient-reported questionnaire on satisfaction with communication of the multiple sclerosis diagnosis. Mult Scler 2014;20:1224-33.

51. Cutrona CE, Suhr JA. Controllability of stressful events and satisfaction with spouse support behaviors. Communic Res 1992;19:154-74.

52. Greco M, Cavanagh M, Brownlea A, et al. Validation studies of the doctors' interpersonal skills questionnaire. Educ Gen Pract 1999;10:256-64

53. Solari A, Mattarozzi K, Vignatelli L, et al. Development and validation of a patient self-assessed questionnaire on satisfaction with communication of the multiple sclerosis diagnosis. Mult Scler 2010;16:1237-47.

54. Solari A, Martinelli V, Trojano M, et al. An information aid for newly diagnosed multiple sclerosis patients improves disease knowledge and satisfaction with care. Mult Scler 2010;16:1393-405.

55. Giordano A, Lugaresi A, Confalonieri P, et al. Implementation of the 'Sapere Migliora' information aid for newly diagnosed people with multiple sclerosis in routine clinical practice: a late-phase controlled trial. Mult Scler 2014;20:1234-43.

56. Jones J, Hunter D. Consensus methods for medical and health services research. BMJ 1995;311:376-80.

57. Fink $A$, Kosecoff J, Chassin M, et al. Consensus methods: characteristics and guidelines for use. Am J Public Health 1984;74:979-83.

58. Crabtree BF, Miller WL. Doing qualitative research. London, UK: Sage Publications, 1992

59. Silverman D. Interpreting qualitative data. London, UK: Sage Publications, 1993.

60. Mc Cracken G. The long interview. London, UK: Sage Publications, 1988.

61. Snowdon C. Qualitative and mixed methods research in trials. Trials 2015;16:558.

62. Tong A, Sainsbury P, Craig J. Consolidated criteria for reporting qualitative research (COREQ): a 32-item checklist for interviews and focus groups. Int J Qual Health Care 2007;19:349-57.

63. O'Brien BC, Harris IB, Beckman TJ, et al. Standards for reporting qualitative research: a synthesis of recommendations. Acad Med 2014;89:1245-51.

64. Eysenbach $\mathrm{G}$. Improving the quality of Web surveys: the checklist for reporting results of internet E-Surveys (CHERRIES). J Med Internet Res 2004:6:e34.

65. Washington AE, Lipstein SH. The patient-centered outcomes research institute-promoting better information, decisions, and health. N Engl J Med 2011;365:e31.

66. Joint report on health care and long-term care systems \& fiscal sustainability.volume 2 country documents (re-edition). Institutional Paper 2016;037 https://ec.europa.eu/info/sites/info/files/update joint-report_it_en.pdf.

67. Wisdom JP, Cavaleri MA, Onwuegbuzie AJ, et al. Methodological reporting in qualitative, quantitative, and mixed methods health services research articles. Health Serv Res 2012;47:721-45 